\title{
A Real Time Infrared Imaging System Based on DSP \& FPGA
}

\author{
Babak Zamanlooy, Vahid Hamiati Vaghef, Sattar Mirzakuchaki, \\ Ali Shojaee Bakhtiari, and Reza Ebrahimi Atani \\ Department of Electrical Engineering \\ Iran University of Science and Technology \\ Narmak, 16846, Tehran, Iran \\ \{Babak_Zamanlooe, Vahid_Hamiiativaghef, \\ Ali_Shojaeebakhtiari\}@ee.iust.ac.ir \\ \{M_Kuchaki, Rebrahimi\}@iust.ac.ir
}

\begin{abstract}
The principle, configuration, and the special features of an infrared imaging system are presented in this paper. The work has been done in two parts. First, the nonuniformity of IRFPA is detected using a processing system based on FPGA \& microcontroller. The FPGA generates system timing and performs data acquisition, while the microcontroller reads the IRFPA data from FPGA and sends them to the computer. Afterwards the infrared imaging system is implemented based on DSP \& FPGA. The DSP executes high level algorithms such as two-point nonuniformity correction. The FPGA here performs two functions: the first one is reading the IRFPA video output and sending it to DSP; the second function is reading the corrected data from DSP and sending them to video encoder which converts the digital data to the analog video signal. The experimental results show that the system is suitable for the real time infrared imaging with high quality and high precision.
\end{abstract}

Keywords: IRFPA, Nonuniformity Detection, Nonuniformity Correction.

\section{Introduction}

With the development of Infrared Focal Plane Array (IRFPA) technology the advantages of high density, excellent performance, high reliability and miniaturization have become available in Infrared (IR) imaging systems [1]. At present, acquisition of high quality images has become the key problem of IR imaging systems. Such systems generally need to process mass data in real-time. The processing includes various algorithms such as nonuniformity correction, image segmentation, local characteristics extraction, image de-noising, image enhancement, etc; hence there must be a well integrated high-speed information processing system [2].

Another important problem of infrared imaging systems is fixed-pattern noise (also known as spatial nonuniformity noise) which arises because of the difference in response characteristics of each photodetector in an IRFPA [3], [4]. To solve this problem, photoresponse nonuniformity correction must be applied by software or hardware [5]. 
Because of these requirements, a system has been designed which has the capability of detecting and correcting nonuniformity and displaying high quality infrared image. This imaging system is based on DSP\&FPGA and fulfills the requirements of infrared imaging systems.

Nonuniformity detection system is described in section 2, while infrared imaging system is investigated in section 3 . Next, the experimental results are shown in section 4. Finally, conclusions are drawn in section 5.

\section{Nonuniformity Detection System}

\subsection{Hardware Configuration of the Nonuniformity Detection System}

The schematic diagram of the signal processing system for IRFPA nonuniformity detection based on FPGA \& microcontroller is shown in Fig. 1. This system consists of an IRFPA, a driving circuit, an ADC, a FPGA and a microcontroller.

The IRFPA is an infrared opto-electronic device sensitive to radiation in the 7.7 to 10.3 micrometer spectral region. It includes a high-sensitivity focal plane array formed by photovoltaic Mercury Cadmium Telluride diodes connected to a silicon CMOS readout integrated circuit.

The driving circuit unit provides the necessary signals and supply voltage for IRFPA's proper operation. This board also acts as a buffer so that the ADC board has no effect on IRFPA's video output signal.

The output of IRFPA is an analog signal and the signal processing system is digital, so this analog signal should be converted to digital format first. This is done using ADC, which transforms the analog video signal to digital. In order to be applicable to image data processing with high speed and precision, a 12 bit ADC whose sampling frequency is up to $25 \mathrm{MHz}$ is selected so that a high resolution output of digitized data is obtained.

The FPGA used in IRFPA nonuniformity detection system has two functions. It acts as both synchronization and timing Control Module and harmonizes the other units in the system, including the output circuit unit of the IRFPA and the ADC sample unit. It also acts like a SRAM and stores IRFPA's video output. Another part of the system is a microcontroller that reads the data stored in the FPGA and then sends this data to a computer using the RS232 standard.

\subsection{Software of the Nonuniformity Detection System}

The software of the FPGA has been written using verilog hardware description language. The written software causes the FPGA to store IRFPA output data and also produces the necessary synchronization signals.

The software for the microcontroller has been written using $\mathrm{C}$ language. The written software activates microcontroller serial interface, reads the data stored in the FPGA and sends them to computer. Also a program has been written in MATLAB which reads the IRFPA data from Microcontroller using computer's serial port and saves them in a lookup table. 


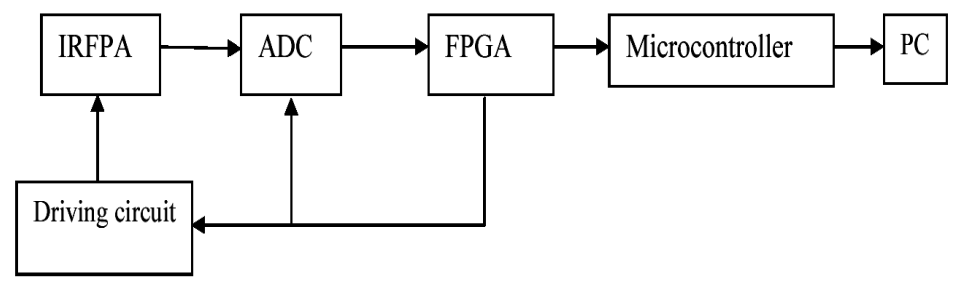

Fig. 1. Schematic diagram of signal processing system for IRFPA nonuniformity detection

\section{Infrared Imaging System}

\subsection{Hardware Configuration of the Infrared Imaging System}

The schematic diagram of the real-time IRFPA imaging system based on DSP \&FPGA is shown in Fig. 2. This system consists of an IRFPA, a driving circuit, an ADC, a FPGA and a high speed DSP.

The ADC transforms the analog output of IRFPA to digital format. The FPGA reads digital video data from ADC and stores them. When one complete frame is read, the DSP reads this data through external memory interface unit (EMIF). The DSP used here is Texas instrument's TMS320VC5510. This DSP achieves high performance and low power consumption through increased parallelism and total focus on reduction in power dissipation. This DSP has an operating frequency of 200 MHZ [6].

The EMIF unit of DSP offers configurable timing parameters so that it can be used as an interface to a variety of asynchronous memory types, including flash memory, SRAM, and EPROM [7]. The FPGA here acts like a SRAM. The DSP reads the video data using EMIF unit and then applies nonuniformity correction coefficients to the data read and corrects them.

After applying nonuniformity correction, the video data is ready for display. But it should be noted that the digital data can not be displayed directly on TV and should be converted to standard television signal. To do this he FPGA reads the corrected data from DSP using host port interface (HPI). The host port interface (HPI) unit of DSP provides a 16-bit-wide parallel port through which an external host processor (host) can directly access the memory of the DSP [8]. The conversion of digital data to standard television signal is done using ADV7177. The ADV7177 is an integrated digital video encoder that converts Digital video data into a standard analog baseband television signal [9].

\subsection{Software of the Infrared Imaging System}

The software written for infrared imaging system consists of FPGA and DSP programs. The FPGA program is written using verilog hardware description language. The written software causes the FPGA to read digital output of ADC and store it like a SRAM, which can be read by DSP. Also the written program causes the FPGA to read the corrected data from DSP using host port interface (HPI) and send them to 


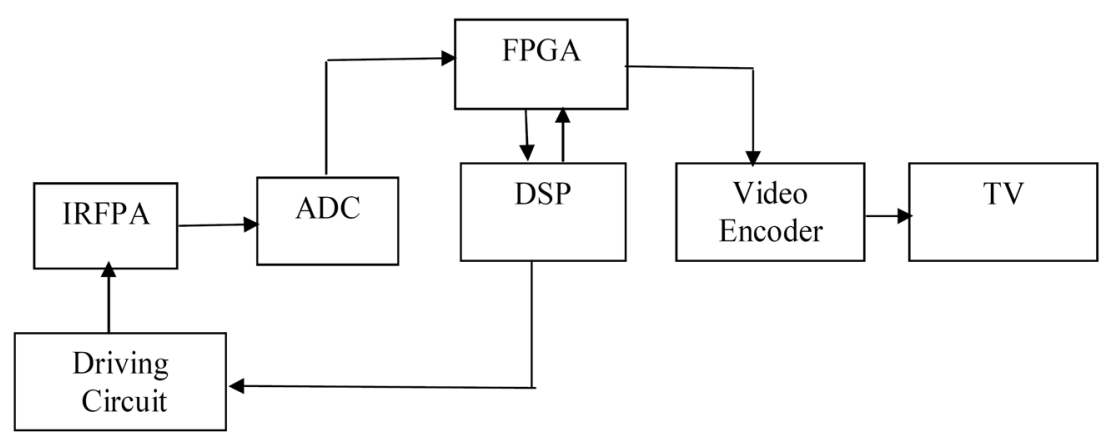

Fig. 2. Schematic Diagram of real-time infrared imaging system

video encoder. The software of DSP is written using $\mathrm{C}$ language. The written software activates EMIF and HPI units of DSP. Also this program applies the nonuniformity correction algorithm to video data.

\subsection{Nonuniformity Correction Algorithm}

The so-called nonuniformity of IRFPA is caused by the variation in response among the detectors in the IRFPA under uniform background illumination. There are several factors causing nonuniformity. The main sources of nonuniformity are: (1) response nonuniformity, including spectral response nonuniformity; (2) nonuniformity of the readout circuit and the coupling between the detector and the readout circuit; and (3) nonuniformity of dark current [5]. Without nonuniformity correction (NUC), the images from the IRFPA are distorted and are not suitable for image formation [10].

There are two main types of nonuniformity correction (NUC) techniques. The first is to calibrate each pixel by the signal obtained when the FPA views a flat-field calibration target (usually a blackbody radiation source) held at several known temperatures, and it assumes the response characteristics of detector are constant temporally; this method is usually called calibration-based correction. The second is to use an image sequence to estimate the correction factors or to estimate the corrected signal directly, this kind of method is based on the scene and requires no calibration of the FPA, and therefore it is called as scene-based correction. Although the latter method is convenient and has developed greatly recently, there exist two disadvantages. The first one is that it does not reveal the correspondence between the signal output and the thermal radiation (or temperature) of the object observed. The other is, for lack of prior information about the FPA, many scene-based techniques are sophisticated and need a procedure to estimate the correction factor, which makes its realization impractical in some real-time systems, especially where the correction needs be implemented by hardware. Consequently, calibration-based NUC methods are still the main compensation method in many IR imaging systems, especially systems used to measure the accurate thermal radiation or temperature of the scene [5]. 
The algorithm used here is a two-point correction method which is a calibrationbased method. In this algorithm detector outputs are assumed to be linear and stable in time, as shown in Fig. 3. Detector output can be expressed as [11]:

$$
S_{i j}(\phi)=K_{i j} \phi+Q_{i j} .
$$

Where $\phi$ represents the incident irradiance on detector $(i, j), S_{i j}(\phi)$ is the output of detector $(i, j)$, and $K_{i j}$ and $Q_{i j}$ are the gain and the offset of detector $(i, j)$ respectively.

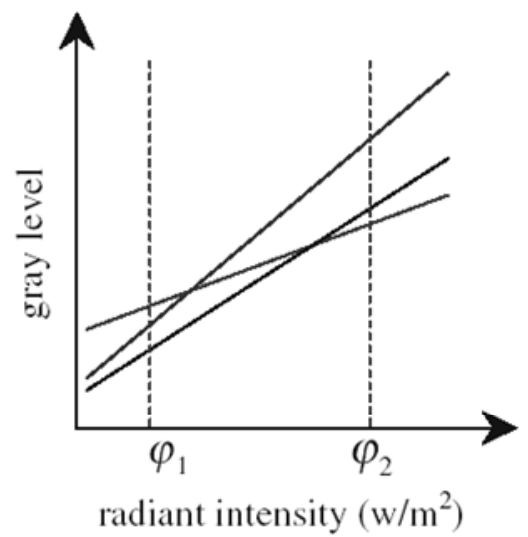

Fig. 3. The linear model of response curve of detector in IRFPA

According to the radiation range of a scene that IRFPA observes, two irradiances $\phi_{1}$ and $\phi_{2}$ are chosen as the correction points, and the detector response data at these two points are recorded using the nonuniformity detection system which was investigated in section 2 . Then the average value of all detectors output $S_{i j}\left(\phi_{1}\right)$ and $S_{i j}\left(\phi_{2}\right)$ in the IRFPA are calculated, respectively.

$$
\begin{aligned}
& \overline{S_{1}}=\frac{1}{N \times M} \sum_{i=1}^{N} \sum_{j=1}^{M} S_{i j}\left(\phi_{1}\right) . \\
& \overline{S_{2}}=\frac{1}{N \times M} \sum_{i=1}^{N} \sum_{j=1}^{M} S_{i j}\left(\phi_{2}\right) .
\end{aligned}
$$

The line determined by $\left(S_{i j}\left(\varphi_{1}\right), \overline{S_{1}}\right)$ and $\left(S_{i j}\left(\varphi_{2}\right), \overline{S_{2}}\right)$, illustrated in Fig. 4, is used as the normalized line for the correction of the response of all pixels. Then the output value $S_{i j}(\phi)$ and its corrected value $S_{i j}^{\prime}(\phi)$ are related as follows: 


$$
\begin{gathered}
S_{i j}^{\prime}(\phi)=\frac{\overline{S_{2}}-\overline{S_{1}}}{S_{i j}\left(\phi_{2}\right)-S_{i j}\left(\phi_{1}\right)} S_{i j}(\phi)+\overline{S_{1}}-\frac{\left(\overline{S_{2}}-\overline{S_{1}}\right) S_{i j}\left(\phi_{1}\right)}{S_{i j}\left(\phi_{2}\right)-S_{i j}\left(\phi_{1}\right)} . \\
i=1,2, \ldots, N . \quad j=1,2, \ldots, M .
\end{gathered}
$$

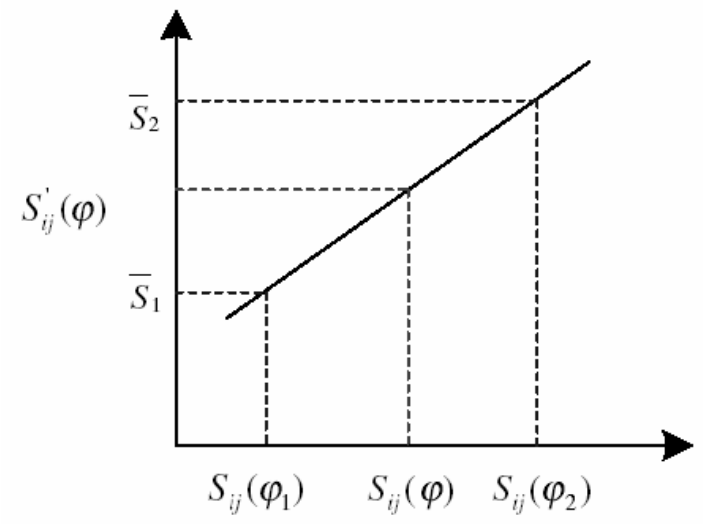

Fig. 4. Sketch map of the two-point correction

The normal two-point NUC based on the linearity model has the advantage of little online computation. IRFPA imaging systems need to process data in real time, therefore this method is selected to correct nonuniformity.

Equation (4) can be written as:

$$
S_{i j}^{\prime}(\varphi)=G_{i j} S_{i j}(\varphi)+O_{i j}
$$

$G_{i j}$ and $O_{i j}$ are the correction coefficients for the gain and offset of the $(i, j)$ detector. $G_{i j}$ and $O_{i j}$ are precalculated and then stored in the FLASH memory unit. When the system is operating, it reads them out of the flash and corrects data it in real time.

\section{Experimental Results}

The performance and capabilities of the IRFPA signal processing system are validated by procedures that connect the image processing system to the IRFPA. The IRFPA is made of Mercury Cadmium Telluride with $4 * 288$ detectors, operating at a frame rate of 100 frames per second. It should be noted that operation of IRFPA at 100 frames per second is due to limitations of imaging system. Results are shown in figures Fig. 5(a) and Fig. 5(b) respectively. Fig. 5(a) is the infrared image before nonuniformity correction. The nonuniformity has distorted the image of the hand. Fig. 5(b) is the infrared image after nonuniformity correction. The imaging quality is greatly higher than raw image. 


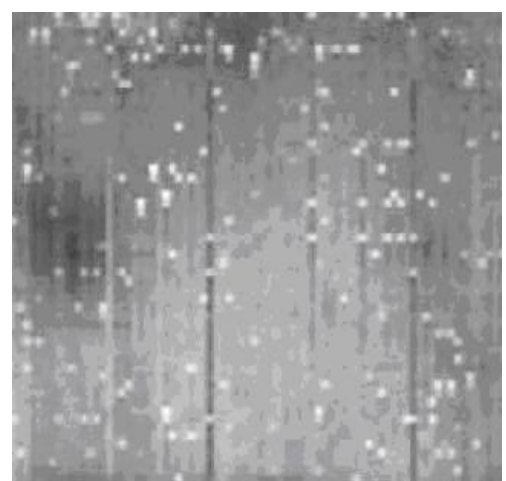

(a)

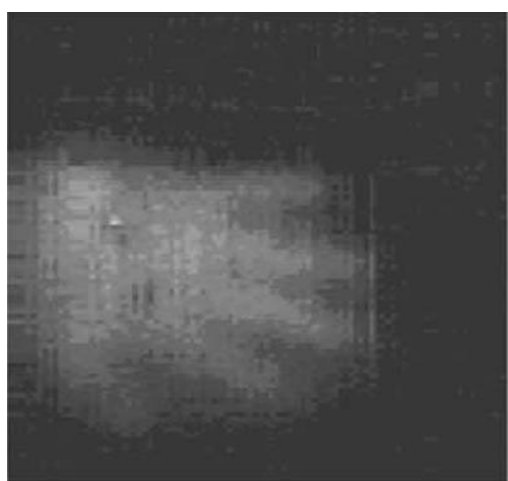

(b)

Fig. 5. (a) Infrared image before nonuniformity correction (b) Infrared image after nonuniformity correction

\section{Summary and Conclusion}

The IR imaging industry is rapidly expanding, thus, the need to improve the performance of processing systems for such applications is also growing.

Nonuniformity detection, correction and displaying high quality infrared image which are done in this paper are the challenging tasks of the IR imaging systems. The proposed IRFPA imaging system has the capability of nonuniformity detection, correction and displaying infrared images and fulfills these complex tasks.

\section{References}

1. Scribner, D.-A., Kruer, M.-R., Killiany, J.-M.: Infrared focal plane array technology. Proceedings of IEEE 79, 66-85 (1991)

2. Zhou, H.X., Lai, R., Liu, S.Q., Wang, B.J.: A new real time processing system for the IRFPA imaging signal based on DSP\&FPGA. Journal of Infrared Physics \& Technology 46, 277-281 (2004)

3. Harris, J.G., Chiang, Y.M.: Nonuniformity correction of infrared image sequences using the constant-statistics constraint. IEEE Transactions on Image Processing 8, 1148-1151 (1999)

4. Milton, A.F., Barone, F.R., Kruer, M.R.: Influence of nonuniformity on infrared focal plane array performance. Journal of Optical Engineering 24, 855-862 (1985)

5. Shi, Y., Zhang, T., Zhigou, C., Hui, L.: A feasible approach for nonuniformity correction in IRFPA with nonlinear response. Journal of Infrared Physics \& Technology 46, 329-337 (2004)

6. TMS320VC5510 Fixed-Point Digital Signal Processors, http://www.dspvillage.ti.com

7. TMS320VC5510, D.S.P.: External Memory Interface (EMIF) Reference Guide, http://www. dspvillage.ti.com

8. TMS320VC5510 DSP Host Port Interface (HPI) Reference Guide, http://www.dspvillage. ti.com 
9. Integrated Digital CCIR-601 to PAL/NTSC Video Encoder, http://www.analog.com

10. Sui, J., Jin, W., Dong, L.: A scene-based nonuniformity correction technique for IRFPA using perimeter diaphragm strips. In: International Conference on Communication, Circuits and Systems, pp. 716-720 (2005)

11. Zhou, H.X, Rui, L., Liu, S.Q., Jiang, G.: New improved nonuniformity correction for infrared focal plane arrays. Journal of Optics Communications 245, 49-53 (2005) 